\title{
Monolithically integrated 1TFT-1RRAM non-volatile memory cells fabricated on PI flexible substrate
}

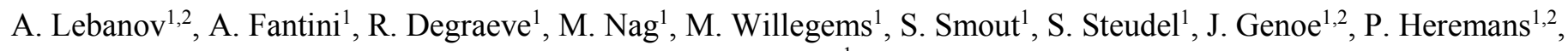 \\ K. Myny ${ }^{1}$ \\ ${ }^{1}$ Imec, Kapeldreef 75, 3001 Leuven, Belgium \\ ${ }^{2}$ KU Leuven, ESAT, 3001 Leuven, Belgium
}

\begin{abstract}
This paper presents an integrated, 1-transistor 1RRAM cell based on amorphous InGaZnO flexible thin-film transistor technology. Both elements of the memory cell have been integrated side-by-side in a monolithic fashion on a flexible polyimide substrate. The thin-film transistor technology shows good uniformity figures, with a minimal channel length of $5 \mu \mathrm{m}$. The non-volatile memory technology is based on TaOx as material for Resistive Random Access Memory. The integrated scalable memory device measures $5 \times 5 \mu^{2}$ and results in a memory window of 7.76 .
\end{abstract}

Keywords-IGZO, metal-oxide, RRAM, memory, flexible

\section{INTRODUCTION}

In recent years, a lot of effort has been put into research on flexible electronics for beyond CMOS applications such as Internet of Things, Radio Frequency Identification (RFID) smart tags and labels, electronic skins. Metal-oxide thin-film transistors (TFTs) on plastic substrates have become a mature technology for backplanes of displays and large-area imagers, and increasingly capture interest for the realization of thinfilm circuits [1, 2, 3]. Recently, more complex RFID tags and even a flexible microprocessor were demonstrated [4]. However, a generic flexible electronics technology platform requires another essential component apart from transistors, namely an electrically re-programmable and non-volatile memory element, preferably integrated into memory banks side-by-side with transistors operating as selecting element.

There are different approaches for the realization of nonvolatile re-programmable memory elements. Many of them have already been demonstrated on flexible substrates [5]. Resistive Random Access Memory (RRAM) shows high potential for integration in flexible platforms due to simplicity of its structure, material deposition techniques suitable for large area applications, low deposition temperatures and good performance. In this work, both TFT as a switch element and TaOx based RRAM as a memory element are fabricated in a single process flow by direct material deposition at low temperatures on a flexible substrate [6]. Thin-film technology, especially when used on plastic substrates, poses various fabrication restrictions compared to the standard CMOS process, such as materials and techniques availability, surface roughness and thermal budget. These restrictions bring challenges that are tackled in this work by synergy of design layout and fabrication methods. We discuss in this work the fabrication technology, including the measurement results and analysis of the monolithically integrated 1TFT-1RRAM memory cells.

\section{EXPERIMENTAL DETAILS}

\section{A. Design}

As previously stated, the focus of this work is to develop an integration route towards an InGaZnO (IGZO)-based TFT and a RRAM memory device. A schematic view on the proposed sample cross-section is depicted in Fig. 1 (a). We chose to use the gate metal (GM) as one of the RRAM contacts, because this leads to several benefits in the integration. It reduces the number of masks and thus simplifies the process. Further, it allows to exploit the flattest surface since all the underlying layers are non-patterned. The 1TFT-1RRAM structures are better balanced in height over the whole wafer as opposed to integration routes in which the memory cell is stacked on top of TFT. However, our integration route requires a careful choice of the gate metal: since it is also the bottom electrode (BE) of the memory cell, it should not scavenge oxygen ions from the memory oxide material. The opposite RRAM contact, top electrode (TE), is covered by anode metal (AM) for routing purposes, achieving electrical connection with the transistor by direct contact to source-drain (S/D). Fig. 1 (b) shows the design layout corresponding to the cross-section presented in Fig. 1 (a), positioning TFT on the left and RRAM cell on the right of the figure. The full stack comprises seven masks aligned on top of each other. For clarity, the colors used in the schematic cross-section match the layer colors in the standard Cadence Virtuoso layout.

\section{B. Fabrication}

The full transistor-memory stack has been developed on a 6-inch polyimide (PI) coated glass carrier wafer by direct material deposition, respecting strict temperature budget limitations $\left(\leq 350{ }^{\circ} \mathrm{C}\right.$ ) imposed by the PI film. The glass substrate is used as carrier during processing, which afterwards can be removed for flexible applications. On top of the PI polymeric substrate, $\mathrm{Si}_{3} \mathrm{~N}_{4}$ barrier layer was deposited followed by an $\mathrm{Al}_{2} \mathrm{O}_{3}$ buffer layer. Subsequently, IGZO semiconductor was deposited and patterned. TFT technology used in this work employs a gate stack comprising $\mathrm{SiO}_{2}$ and $\mathrm{MoCr}$ as metal. Both layers were patterned using one photolithography step. To provide electrical insulation between gate and source-drain metal levels, a $200 \mathrm{~nm}$ thick 


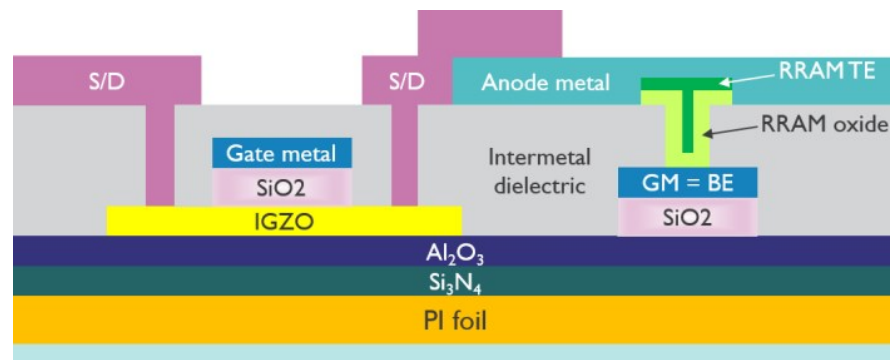

Glass carrier substrate

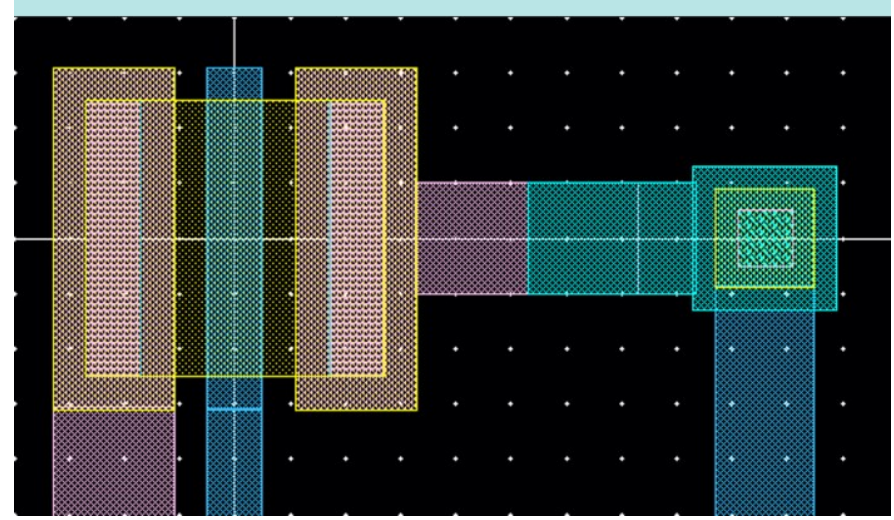

Figure 1. (a) 1TFT-1RRAM cross-section and (b) its corresponding layout

$\mathrm{Si}_{3} \mathrm{~N}_{4}$ called intermetal dielectric (Fig. 8) was deposited. In addition, this layer dopes the IGZO which is not covered with $\mathrm{SiO}_{2}$ making source and drain access regions self-aligned with the gate. The shape and size of RRAM cell are determined by a via in this $\mathrm{Si}_{3} \mathrm{~N}_{4}$ intermetal dielectric layer. After this RRAM via patterning, the memory stack was deposited at room temperature. The RRAM layer was subsequently patterned photolithographically and dry-etched using fluorine-based chemistry. Afterwards, a metal layer comprised of $\mathrm{Ti} / \mathrm{Al} / \mathrm{Ti}$ was sputtered and patterned as AM memory cell contact. The last step of this process flow is providing electrical contact for the source-drain of the transistor. For this purpose, the metal stack $\mathrm{Ti} / \mathrm{Al} / \mathrm{Ti}$ was chosen, which contacts the semiconductor layer through the dedicated areas etched in the intermetal dielectric.

\section{Electrical Characterization}

Electrical characterization of fabricated TFTs was performed using Cascade Summit 12000 semi-automated wafer-probing system enabling full wafer mapping of TFT transfer characteristics. Measurements of the individual RRAM cells as well as 1TFT-1RRAM devices were conducted using Agilent 4156c parameter analyzer.

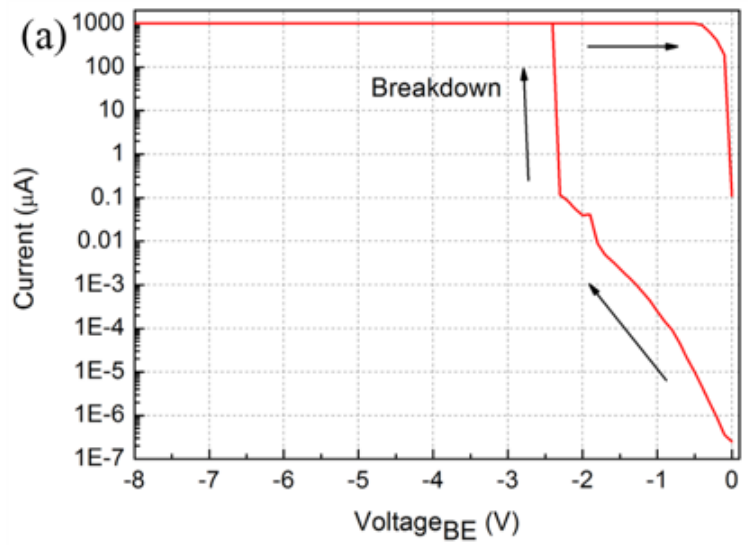

\section{RESULTS AND DISCUSSION}

Fig. 2 (a) plots 180 standard size transistors, which is the total number of repetitive dies on the wafer, while each plot in Fig. 2 (b)-(d) contains characteristics of $\sim 70$ devices corresponding to larger transistors, used in combination with RRAM memory cells. Maximum output currents obtained for different TFT sizes at 5 volts $\mathrm{V}_{\mathrm{GS}}$ and 5 volts $\mathrm{V}_{\mathrm{DS}}$ are as follows: $\mathrm{I}_{\mathrm{ds}} \approx 10 \mu \mathrm{A}$ for $\mathrm{W} / \mathrm{L}=10 / 5, \mathrm{I}_{\mathrm{ds}} \approx 200 \mu \mathrm{A}$ for $\mathrm{W} / \mathrm{L}=250 / 5, \mathrm{I}_{\mathrm{ds}} \approx 500 \mu \mathrm{A}$ for $\mathrm{W} / \mathrm{L}=550 / 5, \mathrm{I}_{\mathrm{ds}} \approx 900 \mu \mathrm{A}$ for $\mathrm{W} / \mathrm{L}=1000 / 5$. TFT onset voltages for all devices is negative and varies around the value of -2 volts.
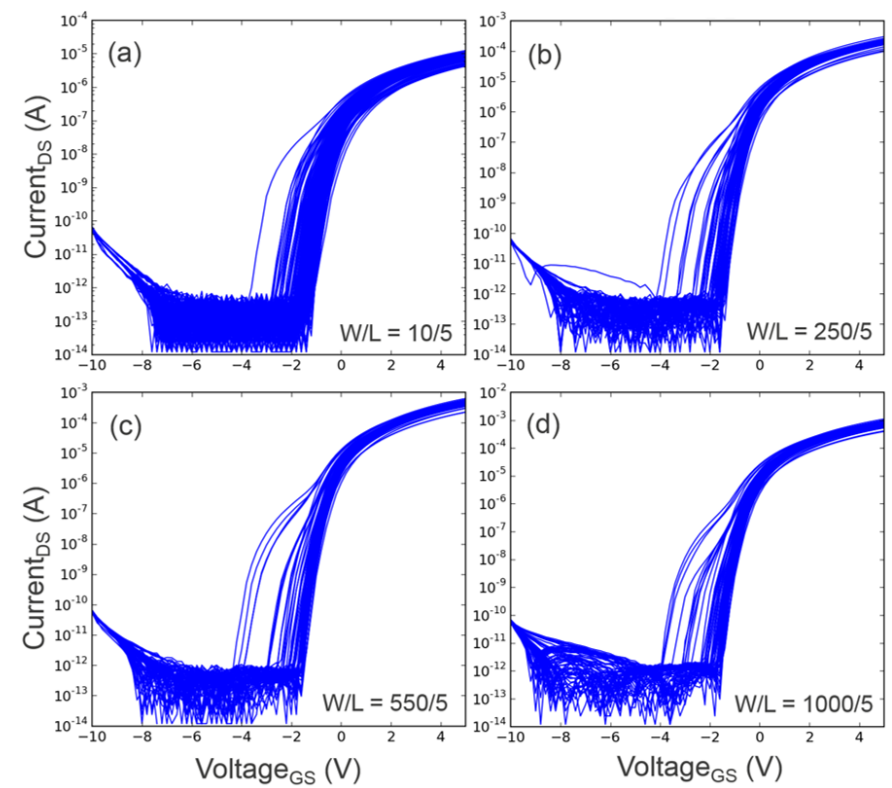

Figure 2. TFT transfer characteristics for the following TFT sizes:

(a) $\mathrm{W} / \mathrm{L}=10 / 5$, (b) $\mathrm{W} / \mathrm{L}=250 / 5$, (c) $\mathrm{W} / \mathrm{L}=550 / 5$, (d) $\mathrm{W} / \mathrm{L}=1000 / 5$

Secondly, the preferential voltage polarity across the RRAM memory cell during the forming step was determined. These measurements are performed by grounding AM and sweeping the voltage on GM RRAM electrode in positive or negative direction. Fig. 3 shows that RRAM breakdown voltage is lower for negative compared to positive voltage polarity. Therefore, we conclude that a negative voltage sweep is preferential for the given memory stack orientation. A potential explanation comes from the fact that the RRAM top electrode internally contacts the transistor while the bottom electrode provides external contact to the power supply, effectively inverting the RRAM stack upside-down (Fig. 1(a)).

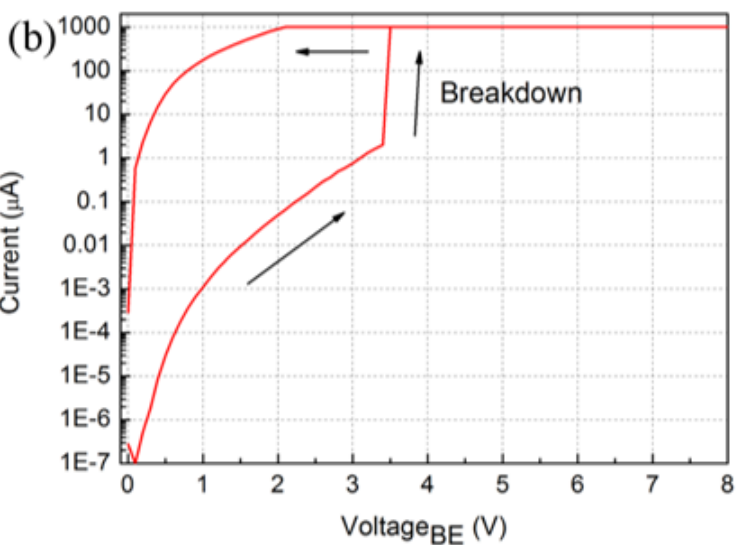

Figure 3. Individual RR fyl cells measurement 
Next, integrated 1TFT-1RRAM structures have been characterized. These measurements contain three sections: forming, reset and set. Knowing that the negative voltage sweep is preferred, as determined in the previous step, voltage is applied across the device in such a way to effectively create a negative voltage sweep at the GM contact of RRAM. Device schematic during the forming step and the obtained measurement curve are presented in Fig. 4 (a). During the forward voltage sweep, the memory cell abruptly switches from a high resistive state (HRS) to a low resistive state (LRS), upon filament formation. This conductive state is retained during the backward sweep of the forming step. Inverting the polarity of the applied dc voltage sweep returns the memory cell into the HRS. This process is known as reset and it occurs in a less steep fashion compared to the forming stage. In the forward sweep of the reset phase, RRAM conductive filament gets disrupted or quenched which limits the current thereby programming the cell into the HRS. The schematic and corresponding measurement curve of the reset process are shown in Fig. 4 (b). After another reversal of the voltage sweep polarity, the memory cell enters set phase and returns from the HRS, established during the reset, back to the LRS. Device schematic followed by a corresponding measurement curve are detailed in Fig. 4 (c). However, shape
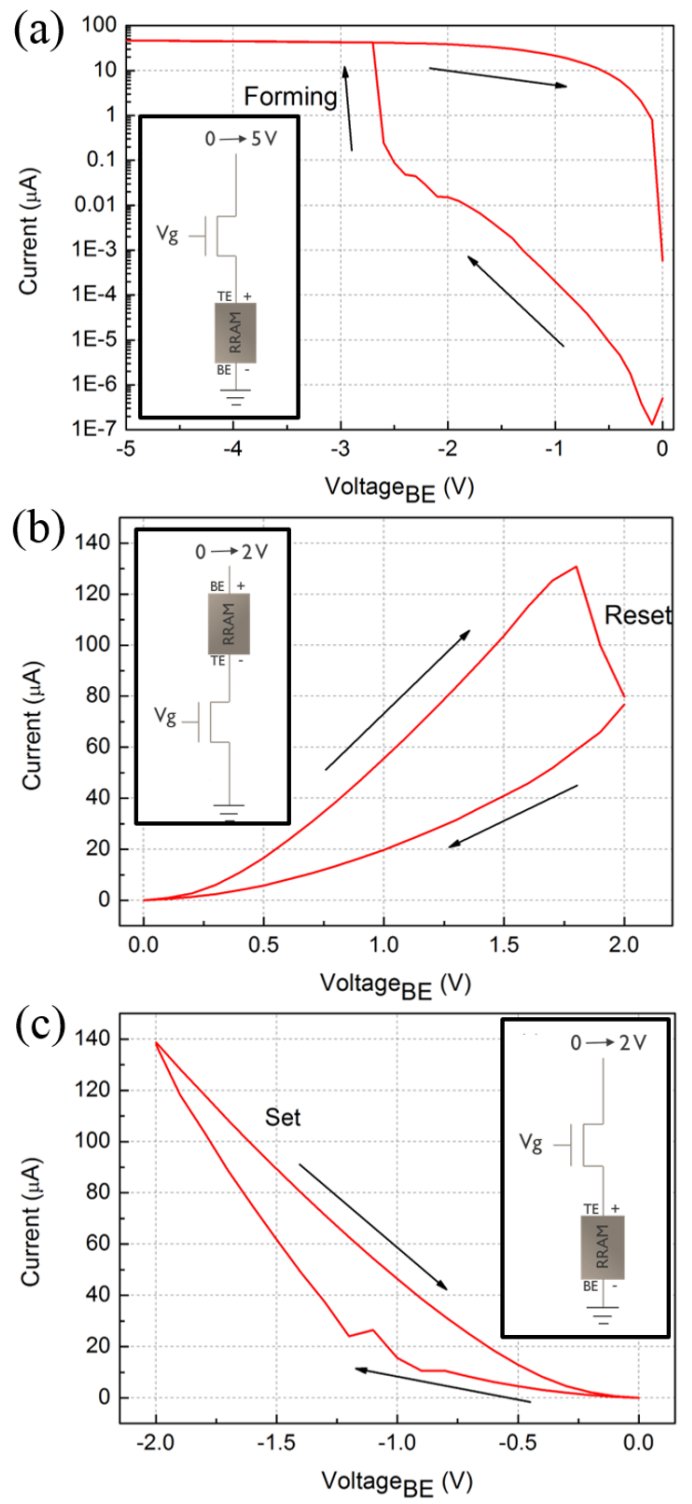

Figure 4. 1TFT-1RRAM measurement conditions: (a) Forming, (b) Reset, (c) Set of the set curve differs from the shape typically reported [7]. This discrepancy stems from the IGZO TFT which, when biased in the way proposed, operates in linear regime and does not reach saturation, which can be explained by high applied gate voltage.

To demonstrate memory cyclability, a device was switched multiple times between HRS and LRS by alternating between set and reset dc voltage sweeps. Fig. 5 presents results of a single cell that cycled through 165 reset and set sweeps. For clarity, a mean value over all the cycles is calculated and presented in red. Additionally, the first, as well as the last setreset cycle are highlighted. The memory window represented

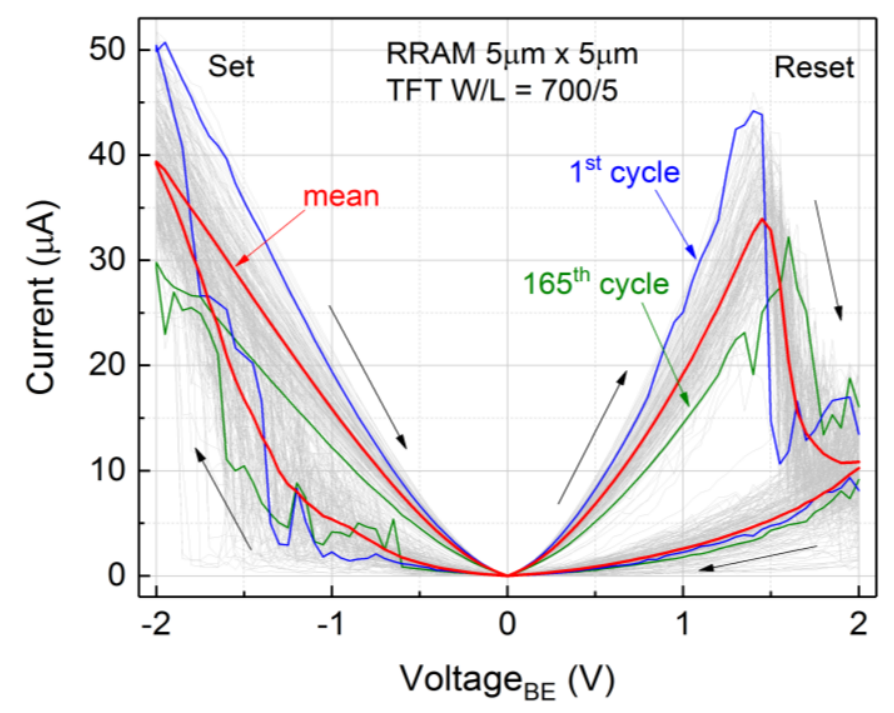

Figure 5. 1TFT-1RRAM device cycling 165 times

as current level ratio between LRS and HRS approximates to the value of 7.76 taken at Voltage $\mathrm{BE}_{\mathrm{BE}}=0.55 \mathrm{~V}$. The current levels between different cycles are repeatable, with HRS showing higher variability compared to the LRS, agreeing with observations previously reported in literature [8]. TFT and RRAM size used in this device are $\mathrm{W} / \mathrm{L}=700 / 5$ and $5 \times 5 \mathrm{~m}^{2}$, respectively. Fig. 6 (a) shows the mean memory window (MW) dependence on the read-out voltage. MW larger than 7 is retained in a broad voltage range, imposing less strict read-out circuitry requirements. Detectable MW is present in each of the 165 set-reset cycles (Fig. 6 (b)), though slightly reducing towards the end.

Microscopic view on 1TFT-1RRAM device is given in Fig. 7 showing a clean structure with straight edges and good alignment. Further optical analysis of the RRAM memory cell structure is performed using Focused Ion Beam Scanning Electron Microscopy (FIB SEM). Fig. 8 shows the side crosssection at the edge of RRAM via. As can be seen, slope of the etched intermetal dielectric is positive, therefore providing deposition of a continuous RRAM stack and ensuring good electrical behavior. Nonetheless, certain adhesion issues between the RRAM and AM layer on the via edge were observed. 

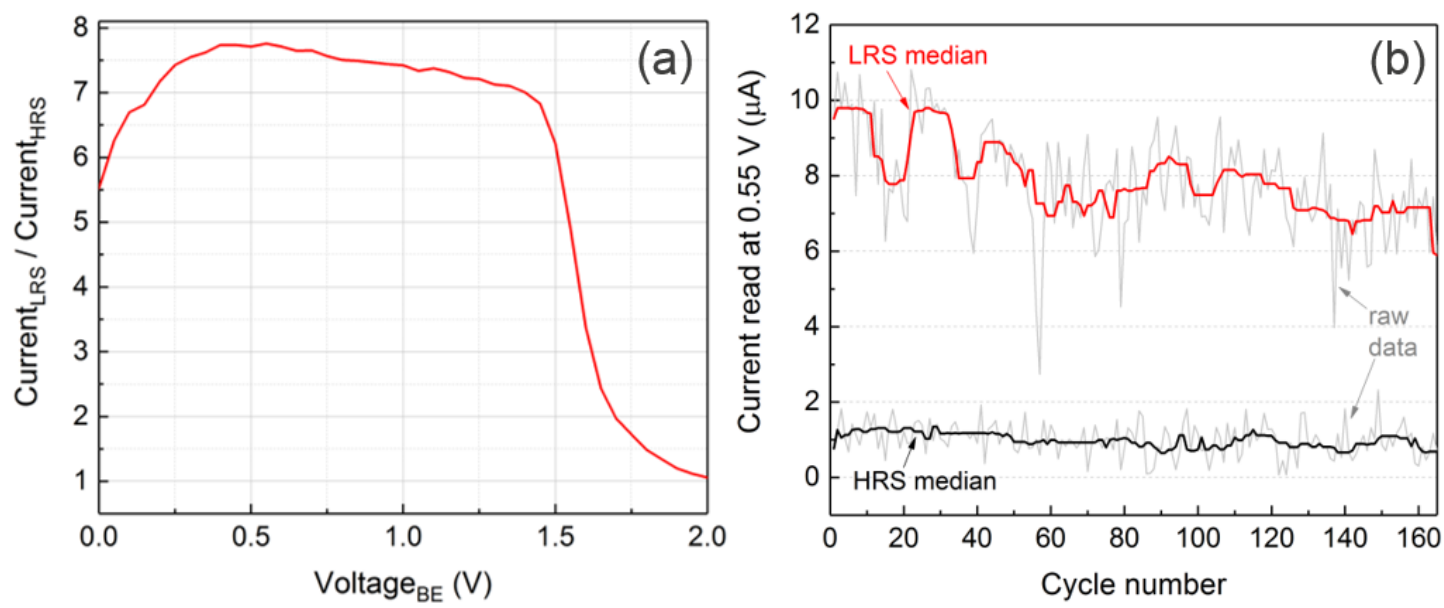

Figure 6. (a) Mean memory window vs read-out voltage, (b) memory window along all cycles

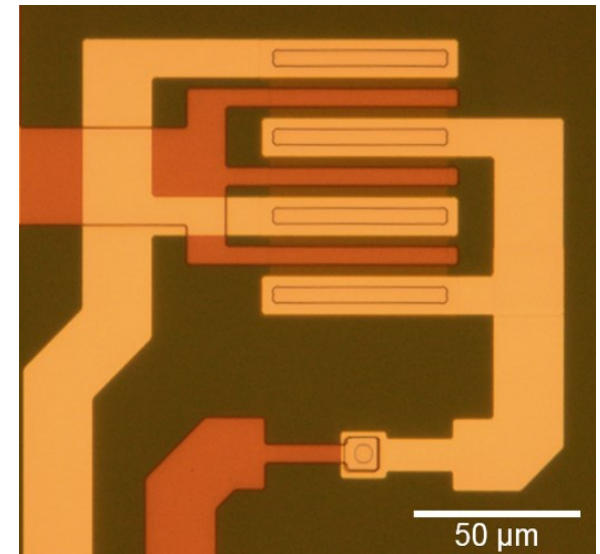

Figure 7. Microscopic view on 1TFT-1RRAM integration

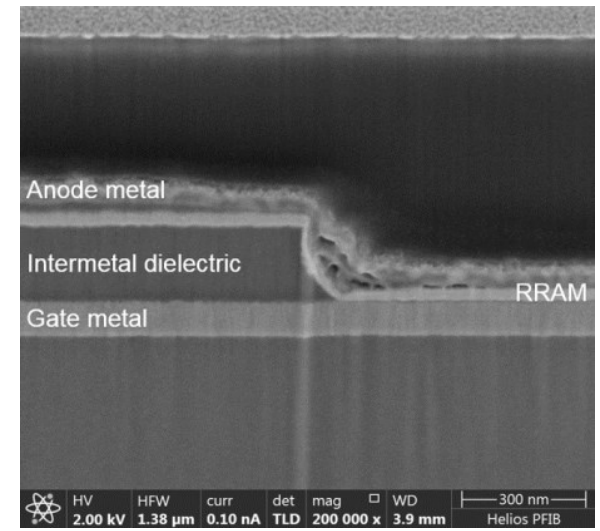

Figure 8. FIB SEM cross-section of RRAM memory cell

\section{CONCLUSION}

In this work, an IGZO-based TFT was chosen as a select transistor for TaOx based RRAM memory cells. We have developed a fabrication process flow which successfully integrates both components side-by-side while promoting simplicity through specially dedicated design layout. The whole device is fabricated in thin-film technology by direct material deposition on flexible substrate, respecting a temperature budget constraint it imposes. Electrical characterization demonstrated proper functioning and provided understanding of developed TFT and RRAM, separately. As the last step, 1TFT-1RRAM structures were tested proving successful integration and cooperation of the select and memory element. The memory cells have been measured for 165 cycles yielding a memory window of 7.76. These values will enable direct read-out by TFT circuits.

\section{ACKNOWLEDGMENT}

This work has received funding from the European Research Council (ERC) under the European Union's Horizon 2020 research and innovation program under grant agreement No 716426 (FLICs project).

\section{REFERENCES}

M. Nag et al., "Low-temperature formation of source-drain contacts in self-aligned amorphous oxide thin-film transistors," J. Inf. Disp., vol. 16, no. 2, pp. 111-117, 2015.

[2] J. S. Yoon et al., "55-inch OLED TV using optimal driving method for large-size panel based on InGaZnO TFTs," Dig. Tech. Pap. SID Int. Symp., vol. 45, no. 1, pp. 849-852, 2014.

[3] C. Zhao, and J. Kanicki, "Amorphous In-Ga-Zn-O thin-film transistor active pixel sensor x-ray imager for digital breast tomosynthesis," Med. Phys., vol. 41, no. 9, p. 91902, 2014.

[4] K. Myny, "The development of flexible integrated circuits based on thin-film transistors," Nat. Electron., vol. 1, no. 1, pp. 30-39, 2018.

[5] M. Ghoneim and M. Hussain, "Review on Physically Flexible Nonvolatile Memory for Internet of Everything Electronics," Electronics, vol. 4, no. 3, pp. 424-479, 2015.

[6] T. Inose, S. Aritome, R. Yasuhara, S. Mishima, and K. Takeuchi, "Study of error repeatability and recovery in 40nm TaOx ReRAM," Eur. Solid-State Device Res. Conf., pp. 10-13, 2017.

[7] N. Sedghi et al., "The role of nitrogen doping in ALD Ta2O5and its influence on multilevel cell switching in RRAM," Appl. Phys. Lett., vol. 110 , no. 10 , pp. $1-6,2017$.

[8] R. Degraeve et al., "Hourglass concept for RRAM: A dynamic and statistical device model," Proc. Int. Symp. Phys. Fail. Anal. Integr. Circuits, IPFA, no. 2, pp. 245-249, 2014. 